九州大学学術情報リポジトリ

Kyushu University Institutional Repository

\title{
Ueber Die Einwirkung Von Eisen Und Hydroperoxyd Auf Polysaccharide
}

\section{Yamafuji, Kazuo}

Agrikulturchemischen Institut der Kyushu-Universität

Urakami, Munehiko

https://doi.org/10.5109/22638

出版情報 : 九州大学大学院農学研究院紀要. 9 (4)，pp.333-339，1950-11. Kyushu University バージョン：

権利関係 : 
Journal of the Faculty of Agriculture, Kyüshū University, Vol. 9, No. 4

November 30,1950

\title{
UEPER DIE EINWIRKUNG VON EISEN UND HYDROPEROXYD AUF POLYSACCHARIDE
}

\author{
Kazuo YamafujI und MunehiKo UraKami
}

Hydroperoxyd wird nach unserer Theorie fast immer in allen Zellen als Folge der Sauerstoffatmung gebildct. Das gebildete Peroxyd wird sofort durch Katalase gespaltet, und es ist möglich, die dabei frei gewordene Encrgic zu den Lebensvorgängen zu dienen. Ausserdem wirkt es auch als Oxydationsmittel. Obwohl das Wasserstoffperoxyd solche physiologisch günstigen Funktionen hat, ist es an sich ein Zellgift. Findet daher eine Anhäufung von Hydroperoxyd in der Zelle statt, so übt es auf die Stoffwechselprozesse ungünstige Wirkung aus. Als ein Beispiel dafür können wir die Tatsache anführen, dass das Wasserstoffperoxyd bei der Virusbildung eine wichtige Rolle spielt. Nämlich erfolgen nach unseren Untersuchungen eine Deraturierung sowie Polymerisation des Nucleoproteins, die für die Neubildung und Vermehrung des Virus unerlässliche Vorgänge sind, in den meisten Fällen unter katalytischer Wirkung des Hydroperoxyds (1). Wenn Hydroperoxyd wirklich als Katalysator bei der Eiweisspolymerisation wirken kann, so liegt es nahe anzunehmen, dass das Peroxyd bei der Bildung der Polysaccharide in den Pflanzenkörpern beteiligt ist. In den grünen Zellen wird wahrscheinlich, wie wir früher gezeigt haben (2), eine Hydroperoxydbildung auch durch Photolyse von Wasser bewirkt. Die so starke Bildung des Wasserstoffperoxyds in den chlorophyllhaltigen Geweben veranlasste uns zu der Vermutung, dass die Polymerisation oder Kondensation von Formaldehyd, das durch Reduktion von Kohlensäure entstanden ist, unter der Katalysatorwirkung des zugleich gebildeten Peroxyds vor sich geht. Da es aber sehr schwer ist, die synthetischen Prozesse im lebenden Organismus stufenweise zu verfolgen, haben wit hicr die umgekehrten Reaktionen, die Zersetzungen der Poly. 
saccharide, untersucht. Die Versuche ergaben, dass durch die kombinierte Wirkung von Eisen und Wasserstoffperoxyd sogar bei der Cellulose eine Zerlegung des Moleküls bewirkt wird, und zwar bei der Stärke sowie dem Xylan eine Bildung der Monosen.

\section{Cellulose}

Cellulose ist eine von den beständigsten Natursubstanzen, und Reagenzien, die eine Zersetzung von Cellulose hervorrufen, können stärker auf andere Polyosen einwirken. Daher wurden zunächst einige Versuche über die Cellulosezerlegung durch Wasserstoffperoxyd angestellt. Die Wirkung des Wasserstoffperoxyds verknüpft eng mit seinem Spaltungsvorgang; die Geschwindigkeit der Hydroperoxydspaltung durch Katalase ist aber zu gross, um die aktiven Spaltprodukte genügend lange mit den Stoffen im Reagensglas reagieren $z u$ lassen. Wir haben jedoch in einer früheren Mitteilung nachgewiesen, dass Methylenblau unter Zusammenwirken von Wasserstoffperoxyd und Eisen reduziert wird (3). Die Methylenblauentfärbung wurde dabei durch Wasserstoff hervorgebracht, der bei der langsamen Spaltung des Wasserstoffperoxyds durch Eisen als $Z$ wischenprodukt auftrat. Der Sauerstoff, der bei der Hydroperoxydzersetzung als Endprodukt freigemacht wird, kann die hochmolekularen Substanzen oxydativ zersetzen.

Es wird angenommen, dass eine vollständige Verzuckerung der schwer zersetzbaren Cellulose nur durch die Wasserstoffperoxydwirkung nicht hervorgerufen wird. Unsere Vorversuche, in denen das Zellstoffpulver unter verschiedenen Bedingungen mit einer Eisensalz enthaltenden Hydroperoxydlösung behandelt wurde, zeigten in der Tat, dass keine Bildung reduzierender Zucker selbst nach ziemlich langer Einwirkungsdauer erfolgt. Es ist aber bekannt, dass die relative Länge der Cellulosemoleküle beinahe mit der Viskosität ihrer Lösungen proportional ist. In der vorliegenden Arbeit haben wir deswegen zur Ermittelung des Zersetzungsgrades die Viskositätsmethode angewandt. Als Cellulose wurde schr feines Pulver reines Filtrierpapiers benutzt. Zunächst wurde die Vergleichungsuntersuchung der Wirksamkeit verschiedener Eisensalze ausgeführt (Tabelle I). Nach der bestimmten Zeit wurde die Reaktionsmischung filtriert, der Rückstand zuerst mit Wasser, dann mit Alkohol gut gewaschen und getrocknet. Die hier erhaltene, 
teilweise zerlegte Cellulose wurde in einer Konzentration von $0,5 \%$ in der Schweizerschen Lösung, die nach Gibson dargestellt wurde (4), gelöst und die Viskosität in Wasserstoff mit dem Ostwaldschen Apparat gemessen.

Tabelle I. Reaktionsmischung: $0,5 \mathrm{~g}$ Cellulose $+5 \mathrm{ccm} \mathrm{n} / 10 \mathrm{Fe}-\mathrm{Sal}+10 \mathrm{ccm}$ $\mathrm{n} \mathrm{H}_{2} \mathrm{O}_{2}$. Reaktionstemperatur: $37^{\mathrm{t}}$. Reaktionsdauer: 5 Stunden.

\begin{tabular}{c|c|c|c|c} 
Fe-Salz: & $\mathrm{FeCl}_{2}$ & $\mathrm{FeCl}_{3}$ & $\mathrm{FeSO}_{4}$ & $\left.\mathrm{Fe}_{\mathrm{q}} \mathrm{SO}_{4}\right)_{3}$ \\
\hline Relative Vikosität $\ldots \ldots \ldots \ldots \ldots \ldots \ldots \ldots$ & 1,664 & 1,570 & 1,576 & 1,525 \\
\hline
\end{tabular}

Bei der Hydroperoxydlösung wurden nach dem anfänglichen Zusatz von $10 \mathrm{ccm}$ alle 30 Minuten weitere $5 \mathrm{ccm}$ hinzugefügt. Die Zahlen in Tebelle I lehren, dass die beiden Salze, Chloride und Sulfate von Eisen, eine Fähigkeit haben, unter Mitwirkung von Wasserstoffperoxyd das Cellulosemolekül zu spalten. Bei den Kontrollversuchen, in denen Eisensalze allein oder Wasserstoffperoxyd allein einwirken gelassen wurden, wurde fast keine Viskositätserniedrigung beobachtet. Die relative Viskosität der unbe. handelten Cellulose war 2,105.

Es wurde dann der Einfluss der Wasserstoffionenkonzentration auf derartige Peroxydasewirksamkeit untersucht (Tabelle II). Da wir bei den Vorversuchen gefunden haben, dass der Phosphatpuffer die Viskositätsmessung stört, wurde das $\mathrm{pH}$ der Reaktionsmischung mit verdünnter Natriumhydroxydlösung eingestellt. Die Natur der Cellulose wird aber leicht durch Alkali geändert. Sonach wurde beim $\mathrm{pH}$ über 9 das Glykokollgemisch verwendet.

Tabelle II. Reaktionsmischung: $0,5 \mathrm{~g}$ Cellulose $+2 \mathrm{ccm} n / 10$ Ferrisulfat $+10 \mathrm{ccm}$ $\mathrm{n} \mathrm{H}_{2} \mathrm{O}_{\mathrm{g}}$. Reaktionstemperatur: $37^{\circ}$. Reaktionsdawer: 5 Stunden.

\begin{tabular}{|c|c|c|c|c|c|}
\hline $\mathrm{pH}$ & $\begin{array}{l}\text { Relative } \\
\text { Viskositait }\end{array}$ & $\mathrm{pH}$ & $\begin{array}{l}\text { Relative } \\
\text { Viskositżt }\end{array}$ & $\mathrm{pH}$ & $\begin{array}{c}\text { Relative } \\
\text { Viskosität }\end{array}$ \\
\hline 3,0 & 1,552 & 6,0 & 1,616 & 90 & $\mathbf{1}, 878$ \\
\hline 4,0 & 1,567 & 7,0 & 1,621 & 10,0 & 1,745 \\
\hline 5,0 & 1.605 & 8,0 & 1,583 & 11,3 & 1,725 \\
\hline
\end{tabular}

Beim Mischen von Ferrisulfat mit Natriumbydroxydlösung entsteht ein Niederschlag von Fisenhydroxyd, der ebenfalls auf die Viskositätsermittelung störend wirkt. Deswegen wurde nach 
der Reaktionszeit $1 \mathrm{ccm} 5 \mathrm{n}$ Schwefelsäure zugesetzt und die Cellulose schnell abfiltriert, wodurch der Niederschlag aufgelöst und entfernt wurde. Wie Tabelle II zeigt, findet die Cellulosezersetzung mit Zunahme der Acidität oder Alkalität in grösserem Masse statt. Da der Zerlegungsgrad der Cellulose durch die Salzarten in der Reaktionslösung beeinflusst wird, können wir dic Werte beim $\mathrm{pH}$-Bereich unter 8 direkt mit denen beim $\mathrm{pH}$-Bereich über 9 nicht vergleichen. Bei den Kontrollversuchen wurde festgestellt, dass nur durch $\mathrm{H}$ - oder $\mathrm{OH}-$ Ionen von entsprechenden Konzentrationen keine erkennbare Spaltung des Cellulosemoleküls bewirkt wird.

Ferner wurde die Beeinflussung der Cellulosezersetzung durch die Temperaturerhöhung untersucht (Tabelle III). Die Visłositätsmessung wurde aber wie gewöhnlich bei $30^{\circ}$ durchgeführt.

Tabelle III. Reaktionsmischung: $0,5 \mathrm{~g}$ Cellulose $+2 \mathrm{ccm} \mathrm{n} / 10$ Ferrisulfat + $10 \mathrm{cmn} n \mathrm{H}_{2} \mathrm{O}_{2}$. Reaktionsdauer: 3 Stunden.

\begin{tabular}{l|c|c|c}
\hline Reaktionstemperatur : & $30^{\circ}$ & $45^{\circ}$ & $60^{\circ}$ \\
\hline Relative Viskositat .......................... & 1,573 & 1,529 & 1,466 \\
\hline
\end{tabular}

Die Viskosität der Celluloselösung nimmt mit der Steigerung der Temperatur des Reaktionsgemisches ab. Bei $60^{\circ}$ wird jedoch die Cellulose durch die Einwirkung des Ferrisulfats allein, besonders durch die des Wasserstoffperoxyds, etwas zersetzt.

Die Cellulosezerlegung ist also hauptsächlich auf die Hydroperoxydwirkung zurückzuführen; die Eisensalze wirken in der Regel nur als Katalysatoren. Dementsprechend muss die Viskositätsverminderung von der Wasserstoffperoxydkonzentration abhängig sein. Die Ergebnisse derartiger Versuche enthält Tabelle IV.

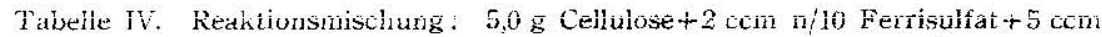
$\mathrm{n} / 20$ bis $\mathrm{n} \mathrm{H}_{2} \mathrm{O}_{2}$. Reaktionstemperatur: $37^{\circ}$. Reaktionsdauer: 3 Stunden.

\begin{tabular}{|c|c|c|c|c|c|}
\hline $\mathrm{H}_{2} \mathrm{O}_{2}$ Konzentration : & $\mathrm{n}$ & $\mathrm{n} / 2$ & $\mathrm{n} / 5$ & $\mathrm{n} / 10$ & $\mathrm{n} / 20$ \\
\hline Relative Viskositat ............ & 1,575 & 1,638 & 1,697 & 1,915 & 1,922 \\
\hline
\end{tabular}

Bei diesen Versuchen wurden wie üblich alle 30 Minuten 5 ccm Hydroperoxydlösung zugesetzt. Die Versuche lassen erkennen, 
dass die Cellulose im Verhältnis zur Hydroperoxydmenge stärker zerlegt wird und weiter, dass die Cellulosezerlegung bei der Hydroperoxydkonzentration unter $\mathrm{n} / 10$ nicht mehr erfolgt.

Wenn die Eisensalze in der Hauptsache als Katalysatoren zur Wasserstoffperoxydspaltung dienen, so genügt eine vehältnismässig kleine Quantität Salze, die Reaktion $z u$ bewirken. Bei den Versuchen in Tabelle $V$ wurden deshalb variierte Mengen Ferrisulfat hinzugefügt.

Tabelle V. Reaktionsmischung: $0,5 \mathrm{~g}$ Cellulose $+0,1$ bis $3,0 \mathrm{ccm} n / 10$ Ferrisulfat + $5 \mathrm{ccm} \mathrm{n} \mathrm{H}_{2} \mathrm{O}_{2}$. Reaktionstemperatur: $37^{\circ}$. Reaktionsdauer: 3 Stunden.

\begin{tabular}{c|c|c|c|c|c|c}
\hline Ferrisulfat in ccm : & 3,0 & 1,5 & 1,0 & 0,5 & 0,3 & 0,1 \\
\hline Relative Viskosität ........... & 1,555 & 1,557 & 1,563 & 1,559 & 1,587 & 1,849 \\
\hline
\end{tabular}

Unter den Versuchsbedingungen in Tabelle $\mathrm{V}$ ist der Ferrisutfatzusatz über $0,3 \mathrm{ccm}$ unnötig. Wahrscheinlich ist der Zerlegung der Cellulosemoleküle durch unmittelbare Wirkung des Eisens sehr gering; die Beschleunigung der Cellulosezersetzung durch Eisensalze beruht mittelbar auf der Förderung der Hydroperoxydspaltung durch dieselben.

Hämoglobin hat ebenfalls eine wasserstoffperoxydspaltende Fähigkeit. Die Viskositätsermittelung wird aber durch Hämoglobinzusatz gestört. Die Cellulose erfuhr keine Zersetzung, selbst als man unter den obigen Bedingungen $10 \mathrm{ccm} \mathrm{n}$ Schwefelsäure anstatt Ferrisulfatlösung hinzufügte. Würde jedoch die Hydroperoxydlösung in der üblichen Weise zur schwefelsauren, eisenfreien Reaktionsmischung zugesetzt, so findet eine ziemlich starke Zerlegung der Cellulose statt.

\section{STÄRKE UND XYLAN}

Wie wir in den oben erwähnten Versuchen festgestellt haben, wird das Cellulosemolekül bis $z u$ einem gewissen Grade durç Zusammenwirken von Eisen und Wasserstoffperoxyd gespaltet. Es ist daher anzunehmen, dass Eisen und Wasserstoffperoxyd auf das Stärkemolekül eine kräftigere Wirkung ausüben. Diesbezügliche Untersuchungen wurden schon von Biedermann (5) und Omori (6) ausgeführt. Doch verfolgten sie den Stärkeabbau nur 
mittels einer Jodfarbenreaktion. Wir haben hier untersucht, ob in solchem Falle eine Zuckerbildung hervorgebracht wird (Tabelle VI).

Tabelle VI. Reaktionsmischung: $10 \mathrm{ccm} 2 \%$ jge Stärkelösung $+20 \mathrm{ccm} \mathrm{m} / 5$ Accetatpuffer $+0,5 \mathrm{ccm} \mathrm{n} / 10$ Ferrisulfat $+5 \mathrm{ccm} \mathrm{n}_{2} \mathrm{O}_{2}$. Reaktions. temperatur: $37^{\circ}$. Reaktionsdauer: 120 Minuten.

\begin{tabular}{c|c|c|c}
\hline $\mathrm{pH}:$ & 3,6 & 5,2 & 6,2 \\
\hline Glucose in $\mathrm{mg} \ldots \ldots \ldots \ldots \ldots \ldots \ldots \ldots \ldots \ldots$ & 13 & 8 & 6 \\
\hline
\end{tabular}

Da das Wasserstoffperoxyd auf die Zuckerbestimmung nach Bertrand störend wirkt, wurde die Reaktionsmischung nach der Reaktion mit $\mathrm{m} / 15$ sekundärem Natriumphosphat neutralisiert und dann mit kristallisierter Katalase aus der Rinderleber versetzt. Tabelle VI zeigt, dass, wie erwartet, eine Verzuckerung stattfindet. Bei den anderen Zusatzversuchen wurde gefunden, dass bei den gleichen Wasserstoffionenkonzentrationen durch das Hydroperoxyd allein eine geringere Zuckerbildung hervorgerufen wird. Die Versuche wurden ferner mit verschiedenen Pufferlösungen angestellt und ergaben immer ähnliche Ergebnisse.

Schliesslich wurde geprüft, ob durch eine derartige peroxydatische Wirkung auch eine Verzuckerung von Xylan bewirkt wird Tabelle VII).

Tabelle VII. Reaktionsmischung; $5 \mathrm{ccm} 1 \%$ ige Xylanlösung $+10 \mathrm{ccm}$ Pufferlösung $+1 \mathrm{~cm} \mathrm{n} / 10$ Ferrisulfat $+30 \mathrm{~cm} \mathrm{n} \mathrm{H}_{2} \mathrm{O}_{2}$. Reaktionstemperatur :

$37^{\circ}$. Reaktionsdauer: 22 Stunden.

\begin{tabular}{|c|c|c|}
\hline Puffer : & $\mathrm{n} / 10$ Glykokoll-HCl von $\mathrm{pH} 2,3$ & $\mathrm{~m} / 5$ Acetat von $\mathrm{pH} 6,4$ \\
\hline Xylose in $\mathrm{mg}$ & 22 & 17 \\
\hline
\end{tabular}

Die Daten in Tabelle VII sind einige Beispiele aus einer Reihe von Versuchen. Das Xylan ist schwerer zersetzbar als die Stärke, ergibt aber beim Zusammenwirken von Eisen und Hydroperoxyd die Pentose.

Die Arbeit wurde durch ein Stipendium des Unterrichtsministeriums gefördert.

(Aus dem Agrikulturchemischen Institut der Kyushu-Universitat in Fukuoka, Japan; 


\section{LITERATUR}

1) K. Yamafuii ut. T. Cho, Ji. agricult. chem. Soc. Japan 20, 411 (1944),--2) K. Yomafuji u. Mitarbeiter, Biochem. Zs. 296, 348 (1938); 298293 (1938); 301, 61 (1939); 303. 60$)(1939) ; 303,260$ (1939); 304, $160(1940) ; 305,354(1940) ; 306,24(1940) .-3)$ K. Yamafuji, K. So u. H. Takaishi, ebenda, 308, 29 (1941,--44) W. Giluson, L. Spencer u. R. McCall, Jl. chem. Soc. 117, 479 (1920).---5) W. Biedernann u. C. Jenerkoff, Biochem. Zs. 149, 309 (1924).-6) T. Omori, Jl. Biochem. 14, $33 !$ (1931). 